\title{
A rapid technique to assess the resting states of the eyes and other threshold phenomena: The Modified Binary Search (MOBS)
}

\author{
RICHARD A. TYRRELL and D. ALFRED OWENS \\ Franklin \& Marshall College, Lancaster, Pennsylvania
}

\begin{abstract}
A technique was developed to automate subjective measurement of the resting states of the eyes. This technique, the Modified Binary Search (MOBS), evolved from the binary search and a commonly used manual bracketing technique. The procedure is mathematically and logically simple, and it requires minimal storage and computation capabilities. Monte Carlo simulations indicate that the MOBS procedure provides more precise measures with fewer stimulus presentations than conventional staircases. It is also relatively insensitive to response errors.
\end{abstract}

Although traditional accounts of accommodation and vergence maintain that both systems relax at optical infinity, current evidence indicates that, when no stimulus is present to activate them, both of these oculomotor systems adjust to an intermediate distance (Owens, 1984). This intermediate bias has numerous perceptual and sensorimotor implications, raising the need for efficient and accurate measurement techniques.

Although objective techniques exist to measure accommodation and vergence, they typically involve infrared recording, which requires sensitive alignment and calibration and is not practical for rapid screening of unpracticed subjects. For this reason, most investigations of the resting states of the eyes have used subjective measurement devices, such as the laser optometer (Hennessy \& Leibowitz, 1972). These devices utilize test targets that have a qualitatively different appearance when presented either nearer or farther than the target value. For example, the laser optometer presents an optical interference pattern containing speckles that may appear to move upward when positioned farther than the subject's accommodative state and downward when positioned nearer than the subject's accommodative state; the target value corresponds to the transition point between upward and downward motion. A manual bracketing technique, similar to clinical methods of visual assessment, has been generally accepted as an efficient method to measure the resting state. In this procedure, the test stimulus is first presented at extreme positions that "bracket" the target value. Over successive presentations, the bracket interval is gradually reduced, thus converging on the final

This research was supported by Franklin \& Marshall College and by Grant EY03898 from the National Eye Institute. The authors gratefully acknowledge Harold Bedell, Ellie Francis, Chris Johnson, and Mark Wagner for their suggestions. Richard A. Tyrrell's current mailing address is Psychology Department, Moore Building, Pennsylvania State University, State College, PA 16802 . Send reprint requests to D. Alfred Owens, Whitely Psychology Laboratories, Franklin \& Marshall College, P.O. Box 3003, Lancaster, PA 17604. measurement. Results obtained from this bracketing procedure are in close agreement with those obtained by more conventional psychophysical procedures (Miller, Pigion, Wesner, \& Patterson, 1983). The bracketing procedure is also used to measure accommodation and vergence responses to adequate stimuli (e.g., Francis \& Owens, 1983; Johnson, 1976).

Despite its advantages, the manual bracketing technique has several potentially serious drawbacks that warrant development of an automated test procedure. Because the manual technique provides no specific test sequence or termination criterion, the experimenter must rely on vague, intuitive criteria to perform the test. This introduces the possibility of unintentional measurement biases, as well as difficulties in control of interstimulus interval (ISI) and replication of test sequences. An automated test procedure may solve these problems without sacrificing the unique flexibility of the manual bracketing technique.

The binary search is an efficient means of searching an ordered array, which, in a manner similar to the bracketing strategy, utilizes information gained with each stimulus presentation to determine the next step of the search. Unlike the bracketing technique, the binary search presents a formal structure on which an automated test procedure can be based. In principle, the binary search requires only $\log _{2} n$ stimulus presentations to identify a target, where $n$ is the number of elements in the array being searched (Lehman, 1977). Thus, only eight presentations should be required to locate a stable target in an array of 256 elements. The search begins by sampling the midpoint of the range of possible values. Depending on the outcome, a "boundary" is established, which, in effect, eliminates half of the array. The midpoint of the remaining range is sampled next, and the process is repeated until the target is located.

Although the binary search is optimally efficient at finding stable targets, it is not suitable for variable targets such as the resting states of the eyes (Johnson, Post, \& 
Tsuetaki, 1984). With the standard binary search, a spurious outcome would result whenever the target drifts outside a previously defined boundary. To be effective, the binary search algorithm must be modified to maintain the integrity of the operating boundaries.

We now report a Modified Binary Search, designated as MOBS, that was designed to combine the efficiency of the binary search with the capability of the bracketing procedure to capture fluctuating targets. This new search technique incorporates the following rules:

1. The sampling range is defined by two boundaries. Each boundary is comprised of a three-element stack, with the top element of each stack representing the current boundary value and the lower elements representing previous boundaries. Initially, all elements of one stack are set to represent one extreme of the range of the measuring system, while the elements of the other stack are set to correspond to the opposite endpoint. One of the two stacks is updated with each presentation.

2. Unless otherwise stated, the stimulus position tested on the next presentation is the value midway between the top elements of the two stacks. Thus, the first presentation will always be in the middle of the measuring system's range.

3. With each response, one of the boundaries is updated. For example, if the response is "nearer," the current stimulus position is placed on top of the stack as the new "far" boundary, and the previous far boundaries are "pushed" down to occupy the second and third positions of the stack. This process of updating the stacks cuts the active test range in half following each response, and information about previous boundaries is saved. This information becomes useful when the target drifts beyond the current boundary.

4. When two consecutive responses are identical, an alternate test is implemented to confirm the validity of the opposite boundary. For example, following two consecutive "nearer" responses, the next presentation tests the position corresponding to the top of the near boundary stack. By testing the opposite boundary, this step confirms that the target remains within the active test range. Otherwise, the opposite boundary is invalid and must be reset as described below.

5. If the target has drifted beyond a boundary (and thus outside the current test range), the invalid boundary is reset to its previous value. This is accomplished by removing the top value from the stack and moving the other values up one step. The bottom element is set to the appropriate endpoint of the measuring system. This event is termed regression.

6. This process continues until two preselected termination criteria are met. The first criterion is that a specified number of reversals have occurred. (A reversal occurs when consecutive opposite responses are collected.) The second criterion is that the last step must be smaller than $5 \%$ of the total measuring range of the system. Although this second criterion is arbitrary, it successfully controls for cases in which the final reversal reflects a large drift of target position. If the previous step was larger than $5 \%$ of the range, the number of reversals is reduced by two and the procedure continues. Upon meeting both termination criteria, the midpoint of the remaining sampling range is taken as the final outcome.

Initial implementations of the MOBS technique were applied to measure the resting states of accommodation and vergence and produced data that agree well with measurements taken by the standard bracketing method. This outcome suggested that MOBS may also provide an efficient means to assess any psychophysical threshold that is based on binary subjective responses (e.g., yes/no, higher/lower). For any application, it is important to know how the efficiency and precision of MOBS compares with those of more conventional methods. To evaluate these characteristics, Monte Carlo simulations were conducted comparing MOBS with outcomes derived from staircase procedures.

\section{SIMULATION 1}

\section{Method}

Monte Carlo simulations were performed on a Franklin Ace 1200 microcomputer (Apple II+ compatible) using Applesoft BASIC. Each execution of a simulation consisted of a search (among 316 possible target positions) for a target that fluctuated normally about a randomly generated mean value. Three levels of variability of target position were used: standard deviations of $0.1,0.5$, and 1.0 units. Each response was determined by comparing the current target with the current stimulus level generated by the MOBS or staircase procedure. Four termination criteria were used: $3,5,7$, and 9 reversals. Efficiency was operationally defined as the number of trials required to complete each execution. Similarly, precision was defined as the magnitude of the $95 \%$ confidence interval for the error of estimating the target's mean value. Relatively small confidence intervals indicate precise estimates. The confidence intervals and mean number of trials per execution were calculated from 50 executions for each condition. In total, each simulation procedure was executed 600 times.

The two-down one-up staircase described by Wetherill and Levitt (1965) was simulated. In short, this procedure differs from a simple staircase in that two consecutive positive responses are required to reduce the stimulus level and only one negative response is required to increase it. The threshold determined by this procedure corresponds to the stimulus level that can be detected $65 \%$ of the time (Wales \& Blake, 1970). The simulation alternated start- 
ing points between the ends of the stimulus range and began with a step size of 10 , which was reduced to a step size of 1 after the first reversal.

\section{Results and Discussion}

Table 1 presents the efficiency and precision data for the two procedures, averaged across the three levels of target variability, for each of the termination criteria. When results are averaged across both termination criterion and target variability, the MOBS procedure required fewer than one-third the number of trials to terminate than did the staircase. This superior efficiency is particularly impressive when one considers that the MOBS procedure was also consistently more precise.

As expected, increasing the number of reversals required to terminate led to greater numbers of total trials in each procedure. Although increasing the number of reversals did increase the precision of the MOBS procedure, this effect was not seen with the staircase.

Figure 1 depicts the mean number of presentations required to terminate for each procedure. Data are depicted as a function of target variability and are averaged across the four termination criteria. The mean number of presentations increased slightly with increasing target variability, indicating that the procedures require slightly more presentations when searching for highly variable targets than when searching for relatively stable ones.

Figure 2 depicts the magnitude of the confidence interval of the estimate, averaged across the four termination criteria, as a function of target variability. The MOBS procedure was more precise (i.e., smaller confidence intervals) than the staircase when searching for variable targets. Although both procedures were more precise when searching for relatively stable targets than for relatively variable targets, the slope of the MOBS function is less than that of the staircase. This indicates that the precision of the MOBS procedure is not as strongly affected by target variability.

\section{SIMULATION 2}

\section{Method}

In determining the $65 \%$ threshold, the two-down oneup staircase does not reflect the most efficient staircase possible. In view of the results from Simulation 1, we decided to compare the MOBS procedure with a more ef-

Table 1

Efficiency and Precision of MOBS and Staircase

\begin{tabular}{cccccc}
\hline & \multicolumn{2}{c}{ Total Trials } & & \multicolumn{2}{c}{$\begin{array}{c}\text { Magnitude of } \\
\text { 95\% C.I. }\end{array}$} \\
\cline { 2 - 3 } \cline { 5 - 6 } $\begin{array}{c}\text { Termination } \\
\text { Criterion }\end{array}$ & MOBS & Staircase & & MOBS & Staircase \\
\hline 3 reversals & 7.3 & 33.0 & & .228 & .240 \\
5 reversals & 10.8 & 42.3 & & .175 & .244 \\
7 reversals & 15.2 & 48.5 & & .137 & .253 \\
9 reversals & 19.5 & 58.7 & & .126 & .248 \\
Mean & 13.2 & 45.6 & & .167 & .246 \\
\hline
\end{tabular}

Note-C.I. = confidence interval.

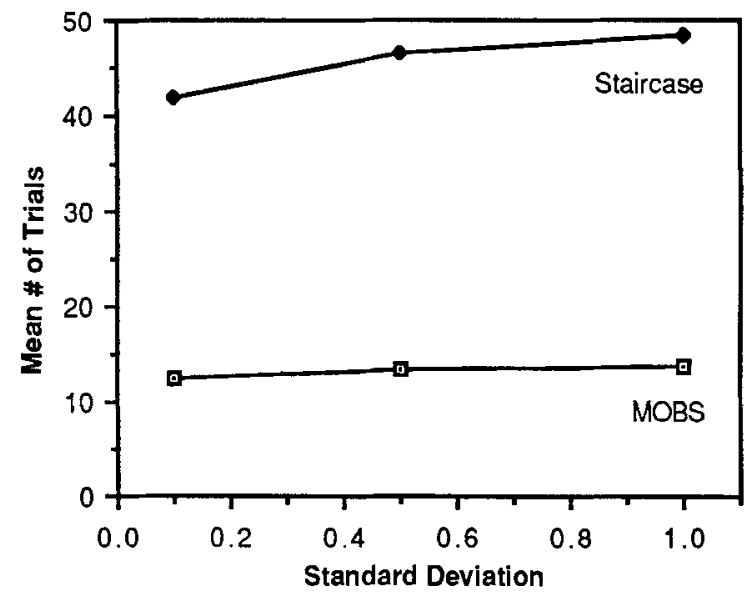

Figure 1. Mean number of trials required to terminate, averaged across the four termination criteria, as a function of standard deviation of target distribution.

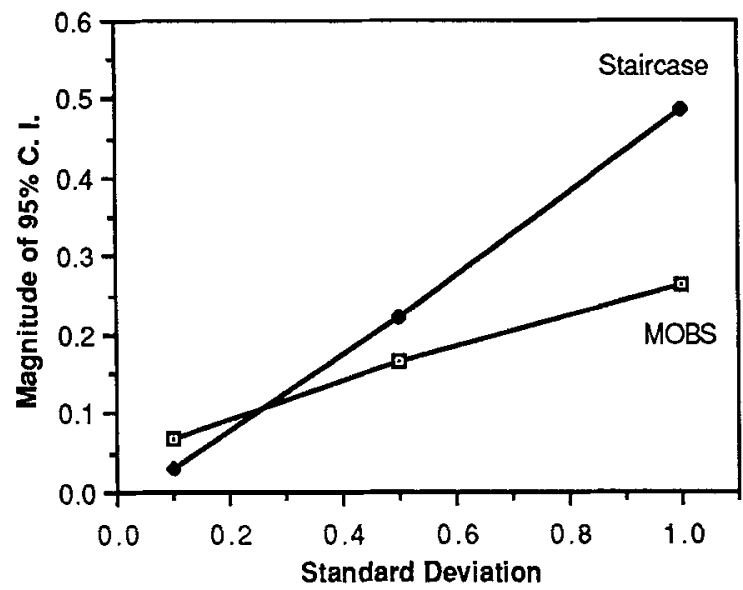

Figure 2. Magnitude of $95 \%$ confidence interval (C.I.), averaged across the four termination criteria, as a function of standard deviation of target distribution.

ficient staircase procedure. Thus, a simple staircase with variable step size was simulated. In this procedure, the stimulus level is reduced with each positive response and increased with each negative response. The threshold determined by this procedure corresponds to the stimulus level that can be detected $50 \%$ of the time. The step size (initially 20) was halved with each response reversal. With the exception of the above changes, the procedure was identical to the previously described staircase. The MOBS simulation was unchanged.

Following Johnson's (1985) investigation of various staircase configurations as applied to automated perimetry, the effects of response errors were also investigated. A response error occurs when a subject unintentionally gives a response inconsistent with his/her perception of the stimulus (e.g., by accidentally pushing the wrong response button). Response errors have been shown to reduce drastically the effectiveness of staircases, which led Johnson to conclude that "there appears to be no 
means for a staircase procedure to recover gracefully from multiple response errors."

Thus, four additional groups of simulations were conducted. In each of the simulations, which included response errors, a $10 \%$ error rate was simulated, with the erroneous responses being positioned randomly.

\section{Results and Discussion}

Figure 3 compares the mean number of trials required to terminate the procedures, averaged across the four termination criteria, as a function of target variability. Both with and without response errors, the MOBS procedure required fewer trials than the staircase procedure. In addition, the effect of the response errors was greater on the staircase than on the MOBS procedure. The mean number of trials required to terminate the staircase procedure increased by 3.1 trials (15\%) when response errors were added to the simulation, whereas the MOBS procedure required an average increase of only 0.9 trials $(7 \%)$.

One explanation for the selective effect of response errors on the staircase is based on the fact that a response error typically causes a spurious reversal to occur, which, in this staircase, causes the step size to be halved. With a smaller step size, additional trials are needed to approach the target position. The MOBS procedure, on the other hand, recovers from response errors relatively quickly. This is accomplished by rechecking the validity of the appropriate boundary (Rule 4) and, if necessary, regressing (Rule 5).

Figure 4 compares the magnitude of the $95 \%$ confidence interval for the estimate of each procedure, averaged across the four termination criteria, as a function of target variability. Without response errors, the MOBS procedure was more precise than the staircase when searching for variable targets. This confirms the results of Simulation 1. When response errors are added to the simulations, however, the difference becomes more strik-

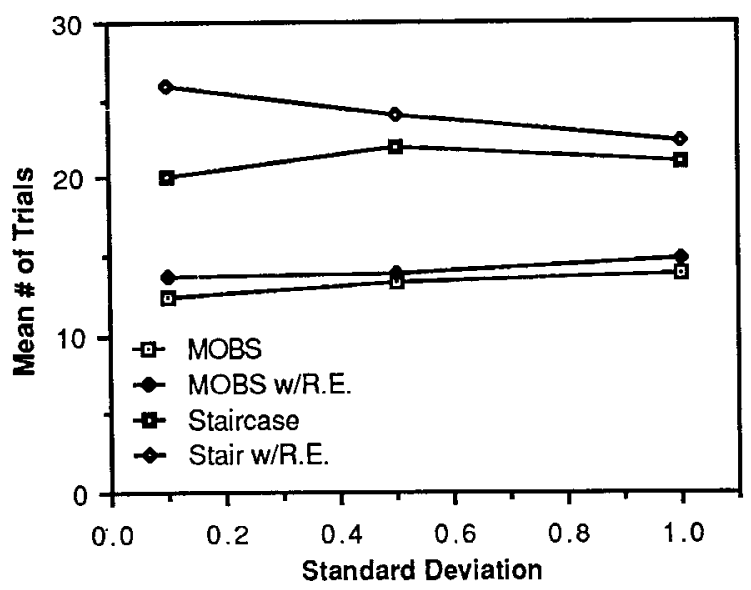

Figure 3. Mean number of trials required to terminate, averaged across the four termination criteria, as a function of standard deviation of target distribution for both procedures with and without response errors (R.E.).

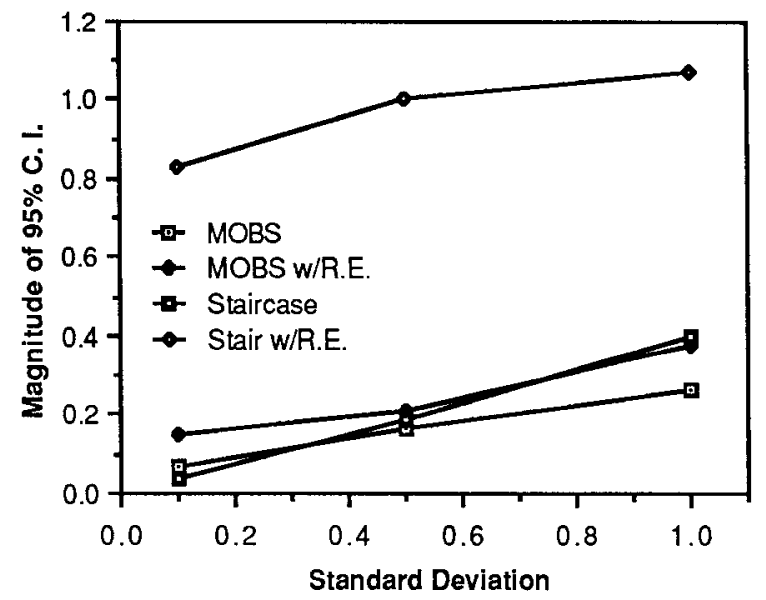

Figure 4. Magnitude of $95 \%$ confidence interval (C.I.), averaged across the four termination criteria, as a function of standard deviation of target distribution for both procedures with and without response errors (R.E.).

ing. The mean confidence interval magnitude for the staircase increased by $372 \%$, whereas the MOBS procedure was not affected as severely, with a $48 \%$ increase in confidence interval size. Again, the staircase is more susceptible to the response errors than the MOBS procedure.

An explanation for this finding is again based on the fact that response errors typically cause spurious reversals to occur. These reversals are not related to the true position of the target value, since the response errors occur at random. Because the staircase procedure uses the mean of all the positions at which reversals occur to determine the final value, the final value will be biased toward the positions where the response errors occurred. The MOBS procedure, on the other hand, does not rely on the reversal positions to calculate the final value, and thus is less susceptible to the response errors.

\section{SUMMARY AND CONCLUSIONS}

The Modified Binary Search procedure, developed originally to measure the resting states of the eyes, appears to be valuable for a wide range of psychophysical applications. Monte Carlo evaluations suggest that this technique provides more precise and efficient estimates of threshold phenomena than do staircase procedures. Moreover, unlike staircase procedures, the MOBS technique is relatively insensitive to response errors. Additional advantages of the MOBS procedure include its mathematical and logical simplicity. The procedure does not require extensive memory or computations, and it is relatively easy to implement on a microcomputer.

\section{REFERENCES}

Francis, E. L., \& OWens, D. A. (1983). The accuracy of binocular vergence for binocular stimuli. Vision Research, 23, 13-19.

HenNESSY, R. T., \& LeIBowitz, H. W. (1972). Laser optometer incorporating the Badal principle. Behavior Research Methods \& Instrumentation, 4, 237-239. 
Johnson, C. A. (1976). Effects of luminance and stimulus distance on accommodation and visual resolution. Journal of the Optical Society of America, 66, 138-142.

Johnson, C. A. (1985). Properties of staircases in automated perimetry. Investigative Ophthalmology \& Visual Science, 26(3), 217.

Johnson, C. A., Post, R. B., \& Tsuetaki, T. K. (1984). Short-term variability of the resting focus of accommodation. Ophthalmic \& Physiological Optics, 4, 319-325.

Lehman, R. S. (1977). Computer simulation and modeling: An introduction. New York: Wiley.

Miller, R. J., Pigion, R. G., Wesner, M. F., \& Patterson, J. G.
(1983). Accommodation fatigue and dark focus: The effects of accommodation-free visual work as assessed by two psychophysical methods. Perception \& Psychophysics, 34, 532-540.

OWENS, D. A. (1984). The resting state of the eyes. American Scientist, 72, 378-387

WALES, R., \& BLAKE, R. R. (1970). Rule for obtaining 75\% threshold with the staircase method. Journal of the Optical Society of America, 60, 284-285.

WETHERILL, G. B., \& LeVitT, H. (1965). Sequential estimation of points on a psychometric function. The British Journal of Mathematical \& Statistical Psychology, 18, 1-10. 